\title{
An Alternative Universe-Scale Analytic Metrology and Related Cosmological Model: An Analysis of Type 1a Supernovae Data With Newly Proposed Distance and Brightness Equations Which, if Valid, Would Eliminate the Need for Changing Expansion Rates of the Universe vis-à-vis Dark Energy
}

\author{
Forrest W. Noble ${ }^{1} \&$ Timothy M. Cooper ${ }^{1}$ \\ ${ }^{1}$ Pantheory Research Organization, 19401A Diamond Ct. Cerritos, California, USA \\ Correspondence: Forrest W. Noble, 19401A Diamond Ct. Cerritos, California 90703, USA. E-mail: \\ pantheory.org@gmail.com, forrest_forrest@netzero.net
}

Received: December 6, 2013 Accepted: January 20, 2014 Online Published: January 26, 2014

doi:10.5539/apr.v6n1p47

URL: http://dx.doi.org/10.5539/apr.v6n1p47

\begin{abstract}
The purpose of this research and study was to help derive and test new theoretical equations to accurately determine cosmological distances and luminosities for all cosmological entities without the need for the dark energy hypothesis. These derived equations were based upon an alternative cosmological model and a study of type 1a supernovae observation data. This paper presents the results of this research and study and the resultant new equations.

A new distance equation is presented in this paper for peer consideration for the first time. It is proposed as an eventual replacement for the Hubble distance formula concerning calculated cosmic distances based upon redshifts. A new "brightness equation" is also offered herein. Its calculations are required to accompany the calculations of the distance equation to determine brightnesses and is proposed as an addendum to the inverse square law of light concerning luminosity calculations for cosmic-redshifted distances. In the subject supernova study these alternative equations are used to show their application and proposed validity concerning calculated distances and observed brightnesses of type 1a supernovae.

The proposed alternative distance equation is very different from the Hubble distance formula since it is directly linear and was derived from an entirely different non-expanding-universe cosmology, tested and refined based upon a study of type 1a supernova data involving the subject applied physics research. Explanations of the alternative cosmological model are presented here for the consideration of their merits. Hopefully these proposed equations will be tested by many others concerning all types of cosmological-distance observations of redshifts requiring distance and brightness-determination calculations, eventually resulting in the serious consideration of the subject cosmological model for reasons discussed in this paper.
\end{abstract}

Keywords: type 1a supernova, alternative cosmology, alternative equations, contradicting dark energy

\section{Introduction}

This paper proposes how an alternative cosmological model, using entirely different distance and brightness equations fit type 1a supernova ( $\mathrm{SN}$, plural $\mathrm{SNe}$ ) data very precisely. This strongly implies that the dark energy hypothesis is the wrong interpretation of supernova data and therefore adds unnecessary complications to the field of cosmology.

To evaluate the advantages of the proposed cosmological model and equations, this paper compares the standard dark energy interpretation of type 1a SN data using the Hubble formula to calculate distances, with the proposed cosmological model which is based upon a Euclidean non-expanding universe (Noble, 2012). After recalculating and plotting these cosmological distances from redshifts using the alternative model equations, there appears to be no indication of the universe's expansion or the acceleration or deceleration of expansion based upon hypothetical dark energy.

The primary purpose of this paper is to present these new equations to accurately calculate cosmological 
distances and luminosities for all cosmological entities, and in this paper and study, for type 1a supernovas. These alternative-model equations are very different from the Hubble distance formula since they were derived from an alternative cosmological model and based upon a study of type 1a supernova (SN, plural SNe) data as presented herein. This paper proposes how this alternative cosmological model, using these entirely different distance and brightness equations, fit SN data very precisely. To evaluate the advantages of the proposed cosmological model and equations this paper compares the standard dark energy interpretation of type 1a $\mathrm{SNe}$ data using the Hubble formula to calculate distances, with the proposed cosmological model which is based upon a Euclidean non-expanding universe model. After recalculating and plotting these cosmological distances from redshifts using the alternative model equations, there is no indication of universal expansion or the acceleration or deceleration of expansion of the universe based upon hypothetical dark energy.

For more than a decade now cosmologists have been investigating numerous possibilities to better explain type 1a SN observations and data as standard candles, with various hypotheses of explanation. As of 2013 some of the most prominent of these hypothesis were the de Sitter invariant special relativity (Wikipedia, De Sitter Relativity, 2013) proposal, the evolving supernova model, the closed dark energy model, the flat dark energy model (Conley et al., 2011), the late-time integrated Sachs-Wolfe effect, dark energy as a cosmological constant, dark energy as Quintessence (Wikipedia, Dark Energy, 2013), other variable expansion models (Wikipedia, Metric expansion of space, 2013), and many other proposals. The models presently being considered by mainstream cosmology, by intent, are consistent with the Big Bang (BB) premise and therefore would not include seemingly feasible but outside-the-box explanations such as the one presented in this paper based upon an alternative cosmological model.

This paper proposes that this alternative cosmological model and its theoretical formulations, properly explain the observations of type 1a SNe and accurately calculate distances and brightnesses based upon their observed red-shifts with completely different results from the Hubble distance formula. The results of these calculations and evaluations of supernova binned data instead support the validity of this alternative cosmological model and its equations calculating distances and brightnesses for all cosmic entities, as well as proposing a new luminosity formula as an addendum to the inverse-square relationship of Electro-Magnetic (E.M.) radiation concerning Luminosity Distances (Wikipedia, Luminosity Distance, 2013). Figure 2 shows that the newly proposed distance and brightness equations produce a relatively straight-line plotted graph of type 1a supernova when compared to the Hubble formula which produces a very curved-line graph.

The proposal that the Hubble formula calculates distances inaccurately is not surprising or unique. Those who support the dark energy hypothesis also assert, based upon their observed luminosity, that both type 1 and $2 \mathrm{SNe}$ with a red-shifts of $z<\sim .5$ (Wikipedia 2013, Accelerating universe), are dimmer and appear to be farther away from us than the Hubble formula calculates. For this reason these observations formed the basis for the dark energy hypothesis.

For all statements concerning "angular sizes", also called "observation angles" and Petrosian angles $\theta$, directly relate to these primary references in this paper: Lopez-Corredoira (2010) and Unzicker (2010). The new distance and brightness formulations used for this supernovae study also support simple explanations for anomalous angular sizes of galaxies as explained by these references.

Some believe that the observed angular sizes of cosmic-redshifted entities are a major problem with Big Bang cosmology. This is because the angular sizes of galaxies and distant entities appear to be too large (anomalous) at low redshift distances, and far too small at the greatest redshift distances based upon their observed angular sizes alone and the studies described in these references. For this reason most observed and calculated angular sizes of cosmic entities are considered anomalous and not easily explained by the Big Bang model. These so-called anomalies are not only predicted by the alternative cosmological model herein, but they are requirements of the alternative cosmological model as explained below. When using the Hubble distance formula to calculate distances to cosmic entities, the ratio of angular sizes to such cosmic redshifted entities has been shown to be closely associated with the relationship $\theta \sim 1 / z$, or $\propto z^{-1}$, ( $\propto$ "proportional to"). Instead when using the alternative cosmological model and its equations to calculate these distances and brightness, the relationship results in $\theta \propto z$, thereby indicating a strictly linear and proportional relationship between cosmological redshifts and distances as proposed by this study of type 1a supernovae data and the resultant derived equations.

\section{Classifying the Alternative Cosmological Model}

The alternative model used herein might be categorized under the broad classifications of Scale-Changing theories, and "variable mass cosmologies" first proposed in the early 1930s. Alternative models at that time were proposed to explain the observed cosmic redshifts by means other than by the expansion of the universe based 
upon galaxies moving away from each other. One of the first of these proposals was made by Paul Dirac when galactic red-shifts were first discovered. He proposed both "the uniform expansion of matter and space". Another proposal was made by Fred Hoyle, Jayant Narlikar (Das, 1998) whereby the diameter of matter accordingly decreases over time by electrons becoming closer to the nucleus of atoms. One of the latter proposals was by Robert Dicke (Wikipedia, Robert H.-Dicke, 2011) directly related to the alternative model proposed herein, whereby in 1957 he proposed that "the cosmological redshift is described by a shortening of measuring rods rather than an expansion of space" (Dicke et al., 2008; Unzicker, 2007) which is the same explanation for the subject cosmological model.

The subject cosmology is also a type of steady-state model but one contrary to an expanding universe, more like the many steady-state proposals before the 1920's. Although Dicke's model is a gravity based model, both models propose that the size of matter relatively speaking, decreases over time, simply that matter is getting smaller as time passes. As to the subject model everything else in the universe would also change in size and magnitude over time but maintain its same relative proportions to matter. This is based on the premise that matter can be used to define everything else in the universe which accordingly would maintain its relative proportions to matter in all time frames. Based upon the alternative cosmology if matter is getting smaller dimensionally at the foundation level of matter such as atomic particles or smaller, then larger matter in the past would have had exactly the same number of atoms in them and be identical to the same matter today only that it would have been relatively larger, but not necessarily larger by direct comparison considering that the foundation particles of matter were accordingly proportionally the same to matter in the past as they are proportional to matter today.

\section{Data Reduction of Type 1a Supernovae}

The type 1a SNe data used for this study and analysis has been organized into averaged groups of adjacent redshifts called bins. There were over 400 separate type 1a SNe observations used for this study based upon publicly available information (Kowalski et al., 2008; Improved cosmological constraints, 2008, 2012), This data was averaged into 17 separate bins. Bin redshift averages were chosen with an effort to evenly space them, leading to averages generally progressing from $z \sim 0.0, z \sim 0.01, z \sim 0.03, z \sim 0.1, z \sim 0.2, z \sim 0.3, z \sim 0.4, z \sim 0.5$, and so forth. Adjacent redshifts of type 1a SNe observations were combined at their average redshifts of their peak brightnesses into separate central redshift "bins."

After this, a secondary unconventional averaging between these bins was conducted: Bin \#1 is represented by the average of supernova data in this bin only, but bin \#2 is an average of bins 1, 2, and 3 averaged together. Bin \# 3 is an average of bins 2, 3, and 4 averaged together and so on. Bin \#17 (the most distant bin) like bin \#1 includes averaged supernova data in this bin range only. As a result of this secondary averaging each separate supernova event is also averaged into adjacent bin(s).

Since type 1a SNe are assumed to be standard candles, the authors of this paper realize that this additional secondary bin averaging additionally smoothes curves and straightens lines by averaging out small brightness differences between supernovas such as possible variations of intrinsic brightnesses, our relative motion within our galaxy, the relative motion of our galaxy, possible human errors influencing the data, etc., but such additional averaging should not disguise telltale non-linear patterns within the data such as those now attributed to dark energy. The Graph, Figure 1, depicts the standard-model graph of supernova with the dark energy interpretation.

For both Figures 1 and 2, $\triangle D M$ designates how much fainter or brighter SNe were than what would be expected in an empty universe model (literally the change $(\Delta)$ in Distance Modulus). Redshifts are the increase in wavelengths over what is observed close by. Dimmer is a positive luminosity number and brighter is a negative number. The graphing is of the variation of type 1a supernovae from expected brightnesses as they relate to observed redshift quantities " $z$ ". 

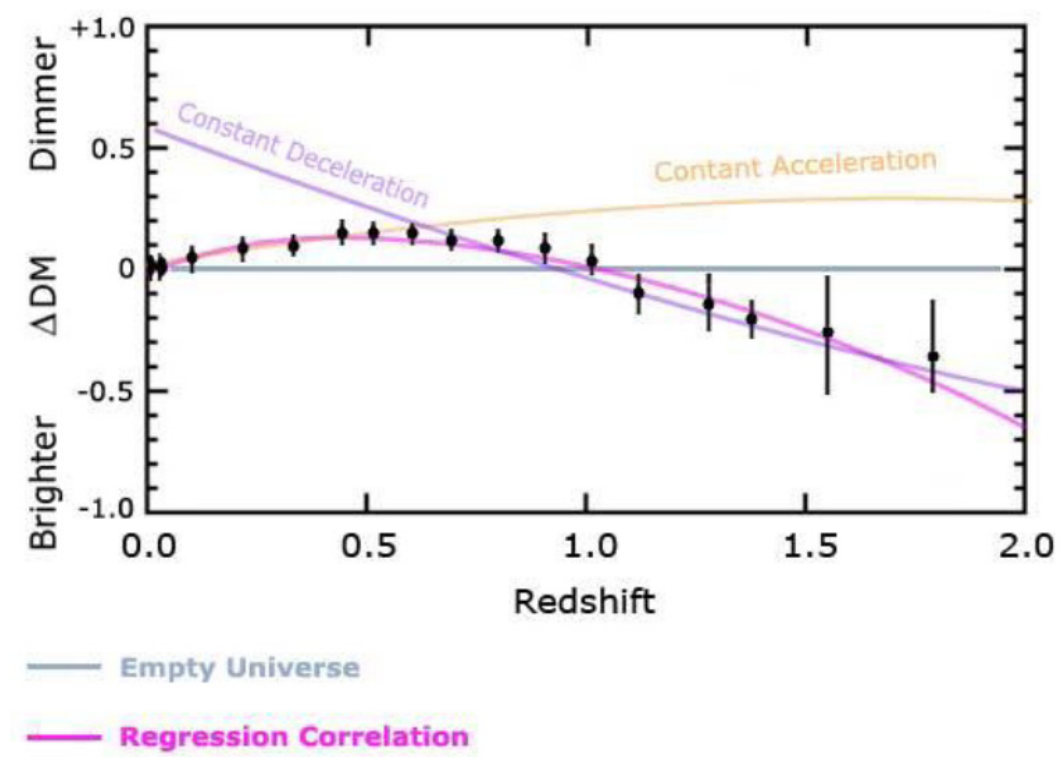

Figure 1. Dark energy interpreted regression lines of type 1a supernovae data

Figure 1 shows the plottings of type 1a supernovae based upon the standard Hubble formula distance calculations and its implications of dark energy. It does not vary a lot from graphs produced by others using generally the same data (Conley et al., 2011). The primary difference between the looks of this graph and other similar plottings using the same data, relates to a secondary averaging of data which was conducted on this data as explained above - which generally would show less linear variations and somewhat reduce the variance from regression lines and curves. The yellowish regression line represents a constant accelerated expansion of the universe, based upon the dark energy hypotheses. The light lavender regression line indicates constant deceleration of the universe starting accordingly over 13 billion years ago and ending at a redshift of about .5 .

(Figure 1 continued). The pinkish colored regression curve represents a best-fit correlation requiring the universe to first decelerate after "Inflation" to a redshift of $\sim .5$, then to accelerate again thereafter to the present day.

This is the general idea of the "Quintessence" version of the dark energy hypothesis, where varying expansion rates of the universe are thought to have taken place for presently unknown reasons. This pinkish line represents the best fit to the observed supernovae data based upon the Hubble formula calculated distances.

\section{Comparison of Hubble Calculated Distances (Blue Parabola) With Distances Calculated Using Equations Derived From the Alternative Cosmological Model (Red Line)}

In Figure 2 the horizontal scale on the bottom represents "redshifts" " $z$ " of type 1a supernovae (where redshifts " $z$ " represent the proportional increase in wavelength, and the entire observed wavelength is equal to $(z+1)$. The vertical scale on the left-hand side represents $\triangle D M$ (the change in brightness) in standard luminosity units which indicates how much fainter or brighter the designated $\mathrm{SNe}$ was compared to what would be expected in an "empty" (Milne) universe model based on the calculated variation from a perfect fit to luminosity distance represented by the centerline 0.0 . 


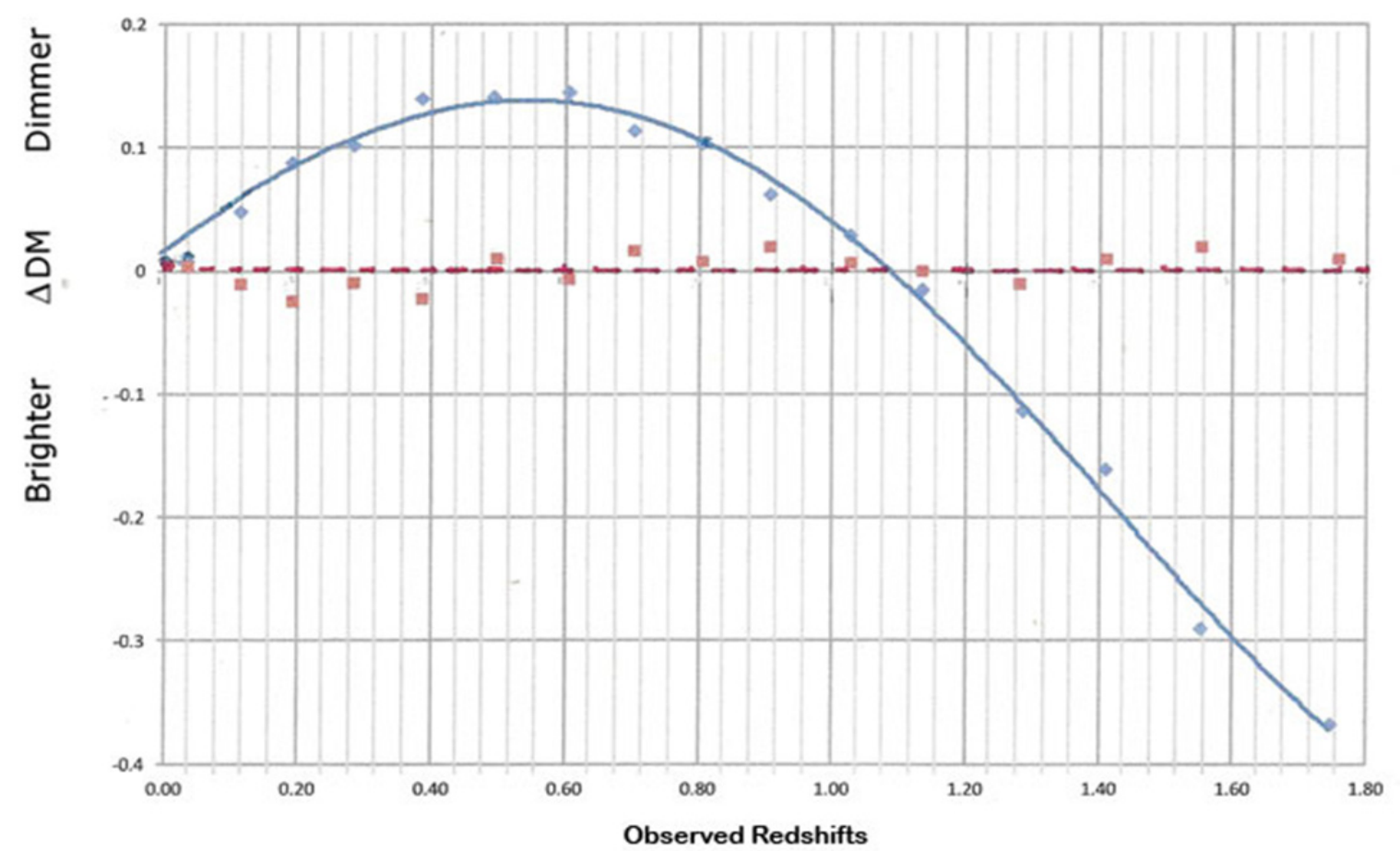

- Standard Model a Alternative Model — Poly. (Standard Model) -..- Linear (Alternative Model)

Figure 2. Comparison of standard and alternative theory brightnesses vs. observed redshift

Two different plots of type 1a SN brightness versus their red-shifts are shown in Figure 2 above. Both plots were developed from published supernovae data (Kowalski et al., 2008, 2012).

The plottings produced by the standard Hubble formula are shown in blue and result in a curved parabolic line with its dark-energy interpretation, as is familiar to cosmologists and most astronomers. This "curved-line" plot was used to support the proposal of the accelerated expansion of the universe and the related "dark energy" hypothesis. Calculations and supernova plottings produced by the alternative equations are shown in red. The result is a "straight-line" plot showing type 1a SNe as true standard candles. The alternative cosmological model is based upon a slow but constant decrease in the size of matter going forward in time (rather than the expansion of space) to explain observed galactic redshifts. The rate of this decrease in the size of matter is calculated to be about $2.2 \%$ per billion years of time which accordingly totally accounts for cosmic redshifts now attributed to expanding space. Cosmological redshifts in the alternative cosmological model are explained by relatively larger matter in the past creating a relatively longer rate of time and longer wavelengths of E.M. radiation. From either perspectives, expanding space or the decreasing size of matter going forward in time, the size of matter relative to space, would decrease over time by the same constant factor.

This alternative plotting, identified as the red binned data bins and red dotted line in Figure 2 red binned data, is what one would hope for when evaluating standard-candle entities using correct distances without the existence of dark energy. When interpreted by the alternative model, the slopes in the standard model supernova graph (Figure 2, blue) are simply based upon an incorrect distance formulation, the Hubble formula.

The focus of this discussion will be on how the alternative formulations of distance and brightness better explains and properly calculates distances and brightnesses of type 1a $\mathrm{SNe}$ observations. It may be more important, however, to realize that these same alternative equations, based upon the subject cosmological model, were formulated to calculate distances and brightnesses of galaxies for all observable cosmic entities including supernovas.

\section{Reviewing the Standard Hubble Distance Formula}

$$
r_{H}=\frac{v}{H_{0}}=\frac{\beta c}{H_{0}}=\left[\frac{(z+1)^{2}-1}{(z+1)^{2}+1}\right] \frac{c}{H_{0}}
$$


The standard Hubble formula shown here as Equation (1), is the basis for the standard model distance calculations shown in Figure 2. The Hubble constant $\mathrm{H}_{0}$ used for this comparison was $66.3375 \mathrm{~km} / \mathrm{s} / \mathrm{Mpc}$, which results in a less exaggerated standard model curve and a flatter alternative model line since $\triangle D M$ brightness determinations were calculated using Hubble-calculated distances, and a somewhat lower cosmological constant produces greater distances and therefore somewhat less variation in the standard model plot from the alternative model. The standard model plot shows that the inflection point in the binned data occurs at a redshift of $z \sim .5$ based upon this plotting (Wikipedia 2013, Accelerating universe); this is often attributed to the Hubble "constant" being considered an average of the universe's expansion rates and asserted to be based upon changes in the force of dark energy.

\section{Continuing Explanations of the Alternative Cosmology}

"The most accurate way to measure redshifts of cosmic entities is by using spectroscopy." Redshifts are determined by both absorption and emission lines of specific observed elements, then comparing the shifted positions and lengths of the spectra as they relate to laboratory spectra of the same elements (Las Cumbres Observatory, 2012). The alternative cosmological model is a diminution of matter model whereby matter very slowly becomes smaller as time progresses.

This model is also a type of steady-state model concerning the observable universe, whereby new matter accordingly is being continuously created and the density of the observable universe remains constant. It is also a model concerning a new concept of relativity in that all aspects of reality, although individually larger in the past, would have had the same relative proportions to matter and therefore past timeframes would have been indistinguishable from the present, if analyses could have been done during those timeframes.

In this paper the explanations of this alternative model primarily relate to how this model better explains type 1a supernovas. There are primarily four observed justifications for this model concerning type 1a supernovae. The first two justifications relate to distances and brightnesses. The combination of the resultant distances and brightnesses have been calculated by alternative equations derived from this model, which seem to be confirmed by observed brightnesses of type 1a supernova without dark energy. The third observed justification relates to the angular sizes observed concerning cosmic redshifted galaxies. Although this does not directly relate to type 1a supernovae, the same distances calculated by the alternative distance equation used for this supernova study also would apply to distances of all cosmic entities. The angular sizes of observed galaxies, concerning the standard model, are considered by many to be an unexplained anomaly of the standard model. The fourth justification concerning observations, relate to a cosmological test concerning the expansion of the universe. This test is called The Tolman surface brightness test, which can be used for all cosmic redshifts and entities.

The Tolman test is based upon the extent of the brightnesses that have been observed based upon distances.

Observations have confirmed those supernovae, galaxies, and all observable cosmic redshifted entities, without additional hypothesis like galaxy evolution or dark energy, fail this fundamental test concerning the expansion of the universe, without the inclusion of additional hypotheses concerning the evolution of the entities being observed. This hypothetical evolution requires observed cosmic entities to have been different in the past from the same entities today. All of the above statements are thought to be totally justified by evidence presented herein, and by other papers referenced in this study. Besides the four types of observations which appear to throw much evidentiary support for the alternative cosmology, other justifications are also mentioned.

\subsection{Explaining the Alternative-Model Details Related to This Study}

The alternative cosmological model proposes that matter becomes smaller in size but proportionally greater in quantity as time progresses. In the past there would have been accordingly fewer individual units of matter than there is now, but over time the density of matter in space would remain the same; as these individual units halve in size, they double in their numbers. These matter units in the future will accordingly be smaller but there will be more of them. For this reason this model is also a type of steady-state model.

If this diminution-of-matter model is correct, what would the red-shift of galaxies whose atoms in the past were exactly twice the size of present-day matter? Take a galaxy equivalent to the Milky Way in every respect, at some epoch in the past; it accordingly would have been twice as voluminous. To us observing it at a redshift of $z$ $=1.5874$, it would appear twice as massive as the Milky Way is now as explained in detail below. Due to proportionality in that time frame, this theoretical galaxy would have been only Milky Way-sized in every way as far as the past epoch and time frame was concerned, having the same relative characteristics as the Milky Way in every respect.

Since atoms can be simply modeled as spheres, the relationship between a spherical atom's volume $V$ and 
diameter $\mathrm{d}$ is shown in Equation (2) below:

$$
d=2 \cdot \sqrt[3]{\frac{3}{4 \pi} V}
$$

If $V=1$ in Equation (2), $d=1.2407$. If $V=2$ in Equation (2), $d=1.5632$. Arranging this into a ratio to represent the reversed diminution of matter, it is shown below in Equation 3 that the diameter of atoms in this past galaxy would have been $2^{1 / 3}(\sim 1.26)$ times larger in diameter.

$$
\frac{d_{2}}{d_{1}}=\frac{2 \cdot \sqrt[3]{\frac{3}{4 \pi} V_{2}}}{2 \cdot \sqrt[3]{\frac{3}{4 \pi} V_{1}}}=\frac{\sqrt[3]{\frac{3}{4 \pi} 2}}{\sqrt[3]{\frac{3}{4 \pi} 1}}=\sqrt[3]{\frac{2}{1}}=\sqrt[3]{2} \approx 1.2599
$$

In this model, the wavelength and amplitude of emitted electromagnetic (EM) radiation is dependent upon the diameter of the atoms emitting it. Therefore, E.M. radiation emitted in the past would have had longer (red-shifted) wavelengths and amplitudes more intense (brighter) by this same factor. Similarly, horizontal distances and velocities relative to the observer would appear to be greater and velocities faster by the same factor of $2^{1 / 3}$ at an observed wavelength of $z+1 \sim 1.5874$. The square root of this wavelength is also $\sim 1.2599$, which again is $2^{1 / 3}$, where $2^{2 / 3} \sim 1.5874$, and $2^{3 / 3}\left(2^{1}\right)=2$.

A "doubling period" in this alternative model is defined as a time interval, when looking backward in time, where the diameter of matter would have been $1.2599\left(2^{1 / 3}\right)$ times its present diameter and twice its present "size" (volume). For three doubling periods past, for instance, matter would have been twice its present diameter, and eight times its present volume.

The perceived recession velocity of distant objects would, according to this model, be directly proportional to the change in the relative size of matter and its proportionality to the measurement of space and its associated velocities. Space is accordingly measured in terms of the size of matter. Standard "rulers" in the past would have been made of bigger atoms and thus past rulers would have been bigger than the same "rulers" today. Standard distances and "space itself" would now be measured as having been larger in the past since measuring "rulers" of space then were also comparatively larger (meters, light years, for instance). Therefore, size relativity over time is based on two factors: one factor would be associated with our perception looking backward in time, regarding the apparent increased size of matter and space, and the other equal factor is related to the difference in the measurement of time now and time in the past.

Since these factors are equal one can calculate the quantity of either factor by taking the square root of the observed red-shifted wavelength. Conversely, the red-shift parameter can be found by squaring either factor as shown below in Equation (4):

$$
z+1=f_{\text {space_size }} f_{\text {matter_size }}=\left(2^{1 / 3}\right)\left(2^{1 / 3}\right)=\left(2^{1 / 3}\right)^{2}=2^{2 / 3} \approx 1.5874
$$

According to this model, the combination of these factors concerning the changing sizes of matter, space, and the length of time, collectively explain presently observed red-shifts and brightnesses, and this can be extended using the same factors concerning velocities. Velocities are defined as distances traveled per unit time. As the distances would have been measured as being relatively larger, time would also have been of a relatively longer duration by the same factor, so the distance traveled per unit of time would still have been the same for a constant velocity (such as the speed of light). Because of this, the speed of light would appear to have been faster by this same factor in the past when compared to today's measuring sticks of distances and time.

\subsection{The Alternative Cosmology: The Theoretical Basis for Formulating Its Equations}

The limit to the distances to galaxies and their ages in the observable universe, according to the alternative model, is solely based upon the capabilities of telescopes rather than the distances and ages allowed by the Hubble formula since the universe accordingly would be vastly larger and probably countless times older than what the Big Bang model proposes.

The equations of the subject cosmological model have no limit concerning the calculable distances and ages of galaxies unlike the BB model, although feasible limits of observation equipment and methods can be estimated concerning the limits of their capabilities.

The alternative-model equations that have been presented in this paper are based upon the theoretical principles 
of the proposed cosmological model, the foundation principle being that matter was relatively larger in the past and slowly gets smaller as time progresses. This accordingly explains the observed cosmic redshifts. With these principles in mind various possibilities were tested against type 1a supernova data until the resultant equations were able to predict observation data. Accordingly all dimensions, measurements, speeds, and time, are accordingly related to, and can be derived from the dimensional changes in the size of matter over time. Constant ratios, like the speed of light, and other primary ratios considered "constants of nature", would accordingly have been measured as having the same values in past time frames as we measure them today.

Hopefully these alternative equations will eventually be tested by the authors and others, against applicable known data, to show their validity, and to possibly refine constants and variables, and/or make adjustments as may be needed. Maintaining consistency with an underlying "comprehensive" theory is a primary consideration to justify any equations in theoretical physics.

\section{Explaining the Derivation of the Alternative Model Distance, Luminosity, and Time Equations}

The alternative model's distance, brightness, and time equations are primarily based upon a single foundation factor which is the square root of observed redshifted wavelengths $(1+z)^{.5}$. The distances and brightnesses calculated by these formulas are, at the greatest distances, much greater than those calculated using the Hubble formula to calculate distances and the inverse square law of light for brightness calculations.

Wavelengths of E.M. radiation are defined as the length between successive crests of a wave. The observed redshifted wavelengths of cosmic entities are accordingly comprised of two equal factors:

One factor creating redshifts would be that matter would appear to have been larger in the past. Larger matter dimensionally would accordingly have produced proportionally longer wavelengths of E.M. radiation for all frequencies.

Distances and space within past time frames would appear to have been larger because smaller yardsticks today would measure greater distances in the past. According to the subject model all ratios would remain constant over time, such as the speed of light for instance. Since velocities are distances traveled per unit of time they are ratios. These velocity ratios can be mathematically represented in the alternative cosmology as $d / t=d_{1} / t_{1}=d(1$ $+z)^{5} / t(1+z)^{.5}$, where $d$ and $t$ are distances and time respectively today, and $d_{1}$ and $t_{1}$ are distances and time in a prior timeframe.

The second equal factor would therefore be time which accordingly measures the rate of changes of matter within timeframes.

Separately each factor of distance and time is equal to the square root of the observed redshift.

\subsection{Deriving the Time-Period Equation}

We will derive an alternative-model time equation that determines intervals of change which we can call "time periods," and "timeframes" for a particular point in time within a time period. This formula is based upon the "doubling periods," described by Equations (2), (3), and (4), determined by the relative changes in the size/measurement of matter and space over time.

The time function, Equation (5), equates modern time with the change in the size of matter over a calculated time interval, where whole numbers represent a complete time period, and decimals a fraction thereof, also seen in Table 1. The 9.966 factor in the formula is a constant which when multiplied by the $\log _{10}$ scale, converts redshift quantities into time interval doubling periods starting from zero, as explained previously concerning Equation (4).

$$
t=9.966 \log _{10}\left[(z+1)^{.5}\right]
$$

The square root of the redshifted wavelength factor is converted into a $\log _{10}$ factor to start time from zero, where zero would represent the present timeframe. All time intervals are positive numbers involving time intervals when looking backward in time. The result is a backward-in-time calculating equation which can equate past time periods with the changing size of matter over time.

The larger the number the farther back in time is being represented. The scale is then adjusted by a constant selected to organize time periods into even integers based upon integers being at doubling periods of matter, as was explained in Equations (3), (4), and (5). A timeframe then would simply be any past point in time.

\subsection{Deriving the "Apparen-Luminosity" Provisional Equation}

Relatively "larger matter" dimensionally in the past would have produced larger, brighter stars, and brighter 
stellar explosions (supernovae), yet in their own timeframes they would have appeared exactly the same as close by supernovae appear to us today.

Based upon matter being larger in the past, supernova and consequently brightnesses would also appear to have been brighter in the past requiring a "brightness-enhancement function", Equation (6), to be used in conjunction with a compatible distance formulation.

$$
\Delta L=\log _{10}\left[\left(\left[\frac{(z+1)^{.5 t}-1}{2}\right] t+1\right)^{2.512}\right]
$$

Explaining Equation (6)

The innermost $(z+1)^{5}$ term (the foundation factor) is related to the constant rate of matter diminution observed as redshifted wavelengths, where matter, space, and the speed of light, by today's measuring standards, would appear to have been greater in size and magnitude in distant timeframes. This foundation factor calculation is based upon the speed-of-light appearing to have been greater in the past, relating to the average calculated speed of light in light years.

The foundation factor is then raised to the power of $\mathrm{t}$, resulting in $(z+1)^{.5 t}$, to take into account the increased size of matter and brightnesses of supernovae progressively looking backward in time, described in Equation (5). For example, after 3 doubling periods backward in time matter would have been eight times larger in diameter and cosmic entities proportionally more energetic. This converts the brightness square root factor $(z+1)^{.5}$, into $(z+$ $1)^{.5 t}$. With the distant observation now changed to our present size-of matter scale, the modified wavelength term then has one subtracted from it (-1) so that only a modified redshift factor remains rather than the whole square root of the wavelength.

This factor is next divided by two to establish an average between the present and past time frames since the speed of light, based upon its measurement, has been changing over its travels proportional to the observed redshift as described above. After this the remaining factor is multiplied times " $t$ ". When looking at the derivation of time, Equation (5), it can been seen that the time period integers calculated by this formula are a proportional analog to the square root of the redshift factor so that by multiplying the results by time would be the same as multiplying it by the square root of the wavelength, then taking the $\log _{10}$ factor to start brightnesses from zero. The constant 9.966 was calculated to delineate time doubling periods into intervals of time.

The result would be two factors based upon the square root of the observed wavelength. The first factor relates to the change in the speed of light over time, and the second factor " $t$ " relates to the perceived changing size of space over time. After combining these two factors together by multiplication, one (1) is then added to the results. This is because the increasing-brightness factor must start at one rather than at zero.

Such an increasing brightness factor could be any number starting from one (1), but not a number less than 1 since that would result in the product that would reduce brightnesses.

Finally the results are then converted to luminosity in the conventional way by taking the observed wavelength factor to a base-10 logarithm, then multiplying the results by 2.512 (Wikipedia, Surface brightness, 2013), or as shown in Equation (6), by taking the wavelength factor to the power of 2.512 and then taking the $\log _{10}$ of that, yielding the same results.

\subsection{Deriving the "Apparent-Distance" Provisional Equation}

The interim distance equation is used for all calculations concerning brightnesses in this study. Although this is not the complete equation its calculations relating to brightnesses are the same as for the completed equation when used in conjunction with the final brightness equation. Similarly, the "interim distance equation", shown below as Equation (7), is derived thus: starting, as usual, with the square root of the observed wavelength.

The basis of the modifying factors of this distance equation is also the speed-of-light from the source to the observer. In this alternative model the speed-of-light was relatively faster in the past by a factor equivalent to the square root of the observed wavelength. The change in the speed of light factor $(z+1)^{5}$ is the foundation factor for this interim distance equation. For a given time period (time of travel), a faster speed of light would represent a greater distance.

Since accordingly the relative speed of light changes over time, the speed of light between the source and the observer would therefore be the average speed of light between the time of its emission and the speed of light now (a lesser speed relatively speaking).

One is added to the other then divided by two. For example: $\left[(z+1)^{.5}+1\right] / 2$; note this is the same as $.5\left[(z+1)^{.5}-\right.$ 
1] +1 . This is turned into a $\log$ factor providing proportional distribution of data while enabling calculated distances to start from zero parsecs rather than one. The constant 21.1695 is multiplied times the logarithm as a fitting factor based on the estimated rate of matter diminution (in particular, it meets the requirements of Equations (3) and (4) above at the doubling periods).

Finally, factor $P_{0}$ is functionally equivalent to Hubble's constant in that it relates wavelength quantitatively to distance quantities (which in this case is a constant of 1958.0), determined and calculated by the supernova data herein given in kilo parsecs.

$$
r_{\text {alt }}=21.1695 \cdot \log _{10}\left(.5\left[(z+1)^{5}-1\right]+1\right) \cdot P_{0}
$$

(where $P_{0}=1958 . \mathrm{Kpc}$ )

Of course the $P_{0}$ factor 1958.0 could be combined with the 21.2946 factor to become a single factor of 41,694.83 designated as $P_{l}$. The last factor is the constant $P_{0}$. Based upon the brightness of the supernova data this constant was calculated to be 1958.0 mega parsecs. Since this constant applies over the entire distance range, it appears to be very accurate since a small variation in its accuracy would become apparent over the full range of supernova charting concerning a variation of brightness inconsistent with supernova data.

In a similar way, the constant factor could be determined as a ratio concerning the changing speed of light and the proposed rate of matter diminution. It also could be directly equated to the Hubble constant based upon supernova observations; if so the constant could be equated with a Hubble constant of about $66 \mathrm{~km} / \mathrm{sec} / \mathrm{Mpc}$, where instead matter would be getting smaller rather than the universe expanding.

This equation produces alternative distances that can be directly compared to the conventional Hubble equation-produced distances. This comparison is then converted to an expected change in brightness, as shown below in Equation (8), where the ratio of the distances is squared based upon the inverse square law of light concerning changing distances, which is applicable in this case. The results are then converted into lumens in the conventional manner.

$$
\Delta B_{1}=\log _{10}\left(\left[\left(\frac{r_{a l t}}{r_{H}}\right)^{2}\right]^{2.512}\right)
$$

Finally, the alternative $\triangle D M$ values are found for plotting the redline plottings of Equation (7), which takes the given observed $\triangle D M$ values (now referred to as $\Delta D M_{H}$ ) to indicate that they are the result of the calculations by the Hubble formula (shown in Figures 1 and 2) and separate them from the concept of $\triangle D M$ in general, and changes them by the "corrected" brightness factor and difference found in Equation (9).

$$
\Delta D M_{\text {alt }}=\Delta D M_{H}-\Delta B_{2}
$$

When comparing the alternative to the conventional model graph at this point, there is no indication in the alternative model, Figure 2 red line, of the accelerated and decelerated expansion of the universe suggested by the conventional curved plot shown in blue in the same Figure. The alternative model red straight-line graphing instead strongly suggests no "expansion" of the universe; instead redshifts are explained in the alternative model by the diminution of matter.

Thus, it is posited that a much simpler explanation than the deceleration and acceleration of a constantlyexpanding universe would be that the standard Hubble distance formula and calculations are simply wrong.

The present interpretation of type 1a supernova data by cosmologists is the reason why the dark energy hypothesis was proposed in the first place. Dark energy has been included in the Big Bang model—now called the Lambda Cold Dark Matter, or Concordance Model—which is presently thought to be the simplest explanation that is in general agreement with observed supernovae data.

However the calculations of the alternative model result in a good fit with the observed luminosity data of type 1a supernova, based upon a flat non-expanding Euclidean universe. It seems apparent that the results produced by the alternative cosmological model with its calculations and resultant plottings, provide a far simpler explanation since its results require the universe to do nothing "special":

The brightnesses/luminosities designated for supernovas as $\triangle D M$ are/were determined by the observing astronomers primarily based upon two factors.

One factor is the inverse square law of light based upon related measuring devices and calculations, called luminosity distances, and the other factor is the reduction of luminosity based upon increased wavelengths resulting from redshifted EM radiation, which is a dimming inverse relationship equal to $1 /(1+z)$. 
The extent of this decreased luminosity is not theoretical. It has been tested and confirmed in laboratories and has generally by known for more than a century. As the wavelength of EM radiation increases the energy of the radiation decreases by this same inverse relationship.

Up to this point we have only included within the distance and brightness equations about $1 / 3$ the magnitude of the entire equation factors independent of the constants. The reason why the entire equations were not used in the graphing calculations of Figures 1 and 2 is that only the factors shown in Equations (6) and (7) vary from the inverse square law of light and therefore would show up as variations of brightnesses in this study which is the basis for the supernova Figures/charts above. The additional factors, explained below, do not show up in observational astronomy because the increased brightnesses produced by larger and brighter matter in the past accordingly would be equally compensated for by equally increased distances, but greatly reduced angular sizes of large galaxies cannot be disguised.

If the additional factors were included in the above calculations the resultant variations of brightnesses would accordingly have been exactly the same. So if these factors of the distance and brightness equations are missing, how could we know of their need or existence and to determine their magnitude other than by the observed angular size?

Based upon Equations (2), (3), and (4) the alternative cosmological model states that type 1a supernovas at a wavelength of 1.5874 , for example, were twice as energetic because the exploding star itself would have accordingly been twice as large dimensionally. However brightnesses could be calculated using either the distance and brightness combinations of Equations $6 \&$ 7, or the combination of Equations 10 and 11 shown below, with both combinations of equations yielding the same brightness results. But separately or combined Equations $6 \& 7$ cannot calculate distances, angular sizes, etc., since they were only formulated for their combined use to make brightness calculations easier.The model itself explains redshifts by the increased size of matter in the past. Increased brightnesses accompany increased size but increased distances would hide increased brightnesses concerning the primary added equation factors shown below.

\subsection{Adding a Final and Primary Factor to Both the Distance and Brightness Equations}

Additional factors are needed for the final distance and brightness equations. This factor is an additional square root of the observed wavelength $(1+z)^{5}$. This additional factor will be added to both Equations (6) and (7).

These addendum factors have not been used or discussed as yet in any past derivations of type 1a supernova. These equations must be combined to determine brightnesses but these added factors to both equations do not effect brightness variations in supernovae, galaxies, or other cosmic entities since increased distances are compensated for by equally increased brightnesses. So these additional factors are not needed for brightness studies like this study where brightness variations are the primary considerations relating to distances.

Instead these added factors result in increased angular sizes for galaxies with redshift values less than .5 , and decreased angular sizes for redshifts greater than this amount (Lopez-Corredoira, 2010; Unzicker, 2010).

\subsubsection{Deriving the Final Distance Equation}

The additional distance factor is: $(z+1)^{5}$, where the entire distance equation becomes

$$
\left.21.2946 \log _{10}\left[.5\left((z+1)^{.5}-1\right)+1\right](z+1)^{.5}\right) P_{0}
$$

The primary factor in the distance equation was explained as being $(1+z)^{.5}$, determined by the relatively changing speed of light, as explained in 7.3. The final factor shown in Equation (10) is also the square root of the observed wavelength. This second factor is based upon the increased relative size of matter in the past as it relates to distances. Using smaller yardsticks today we would measure distances in the past to have been relatively larger, as explained in Equations (3) and (4).

This new factor is greater than the primary factor by an increase in magnitude of half the observed redshift..

\subsubsection{Deriving the Final Brightness Equation}

For the brightness equation the additional factor is also $(z+1)^{.5}$. This factor is squared to determine increased brightnesses based upon the increased area of the observed entity, in this case supernovas. It is a numerator factor in the equation since accordingly increased sizes dimensionally produce brighter observations. This squaring is based upon the increased area of the observed cosmic entity produced by a larger diameter/radius i.e. $\mathrm{r}^{2}$ when looking backward in time. This progressively increasing factor when squared results in a $2 \mathrm{D}$ surface area factor equivalent to the observed wavelength $(z+1)$, where the entire equation then becomes:

$$
\log _{10}\left[\left[\left[\left((z+1)^{.5 t}-1\right)\right) \cdot 5 t+1\right](z+1)^{2.512}\right]
$$


or its equivalent:

$$
\left.2.512 \log _{10}\left[\left[\left((z+1)^{5} t-1\right)\right) \cdot 5 t+1\right](z+1)\right]
$$

Neither of these complete equations was needed or used for this study to determine brightnesses but of brightnesses determined by the combination of these equations would be the same as Equations 6 and 7. Both addendum factors are needed however to determine any and all cosmic distances and to determine angular sizes of cosmic entities based upon cosmic redshifts.

Although the increased brightnesses resulting from these additional factors are hidden in the results by equally increased distances, the angular sizes of the cosmological entities being observed cannot be hidden, as has been discussed. Besides the evidentiary support of this supernova study for the alternative model and its equations with its resultant straight-line plotting, these "hidden factors" are also strong evidentiary support for the alternative cosmological model and maybe the simplest explanation for the presently anomalous observation angles/ angular sizes of galaxies being observed.

In a Euclidean static universe as in the proposed model, angular size vs. redshift dependence should be evident as a direct relationship. Using the Hubble formula to calculate distances, this relationship instead is proportional to $z^{-1}$, in inverse relationship, which should be a primary indicator of the grossly under-calculated distances by the Hubble formula at the highest redshifts. According to the alternative model distances are greater for all supernovae, galaxies and other cosmic entities.

Besides being much farther away and brighter at the farthest distances, this model also predicts that distant galaxies, based upon present distance and brightness calculations, will appear to be smaller and more condensed (angular sizes), and often appear to be more massive than they really were in their own time frames (increased brightnesses).

This cosmological model also predicts that average galaxy appearances, sizes, and types were the same in the past as they appear to us close by and therefore the universe would be much older. The model would assert that we are both lost in space and lost in time, but accordingly past time would not be infinite.

The three chart-forming equations calculate Time, Distance, and Brightness; all of these equations have their basis in the square root of the observed wavelength: $(1+z)^{5}$.

Application: According to the alternative model for instance, distances at a redshift of $z=10$, calculated by the alternative model, are 10 times greater than what the Hubble formula would calculate. The age of the universe accordingly would be many times greater than the Hubble model calculations.

These calculations of the observed angular sizes of galaxies are confirmation of the alternative model calculated distances if no universal galaxy evolution over time exists. This is a huge difference between the two models and their calculations. For distances within the range of the observed type 1a supernova data, distances are about 2.4 times greater using the alternative distance formula than distances calculated by the Hubble formula, but this difference is also very significant.

\section{Other Equations of This Alternative Model}

According to the Standard-Model time dilation formula, time dilation is calculated using the formula $(1+z) t$. For instance at a redshift where $z=1$, (with wavelength of $(z+1=2)$ ), a supernova observation event would accordingly last exactly twice as long.

For the alternative cosmological model the time dilation equation is somewhat different from the equation used by the standard model.

First, time in the past accordingly would have been based upon the square root of the observed redshift $(z+1)^{.5}$ as would be the size of matter and distances as per Equation (4).

In the case of time, however, it would be observed as a slower rate in the past by a factor of $1 /(z+1)^{5}$ in that velocity is distance traveled per unit of time. In this way the speed of light and all related velocities would remain a constant ratio in that the distance traveled would be considered greater, but the length of time would be measured to have been equally longer.

\subsection{Deriving the Alternative Time Dilation Formula}

The alternative equation for time dilation is:

$$
t^{\prime}=f(z, t)=\left(\frac{z}{2}+1\right)(z+1)^{5} t
$$

where $\left(t^{\prime}\right)$ is the extent of the expected time dilation concerning type 1a supernova events, and $(t)$ is the timing of the same event today based upon standard time. All type 1a supernova events with the same redshifts should all 
last about the same amount of time.

This alternative time dilation formula may be the easiest way to evaluate the alternative cosmological model because there would be a slight but observable difference between the length of time dilation of a type la supernova events calculated based upon the standard model and the alternative model which should be observably significant, especially for the most distant type 1a supernovae. The first factor of this derivation is $(z / 2+1)$. This is a half-redshift factor based upon the change in the speed of light. A decreasing rate of time, when looking backwards in time, would be a prediction of the alternative cosmological model. A longer observable time period following a type 1a supernova explosion, we call time dilation.

The second factor in the equation is the square root of the observed wavelengths. This factor is accordingly based upon our perspective of greater measured distances and the increased size of space in past timeframes.

It should be noted that this time dilation equation would also apply to any other possible cosmic observations and events that would last a constant period of time in the present. This difference between the two equations can be seen in Table 1, column a. the standard formula, and column b. the alternative-cosmology formula. 
Table 1. Comparing the larger calculated factors of the alternative cosmology with the standard model calculations concerning: time dilation, distances, angular size, and luminosities, within the entire range of wavelengths/ redshifts

\begin{tabular}{|c|c|c|c|c|}
\hline \multicolumn{2}{|c|}{$\begin{array}{l}\text { a. } \\
\text { Wavelengths }(z+1) \text { of } \\
\text { averaged binned data } \\
\text { (“" } z \text { " value is the redshift } \\
\text { alone) } \\
\text { Also conventional time } \\
\text { dilation formula } \\
t \text { ' }=(z+1) t\end{array}$} & $\begin{array}{l}\text { b. } \\
\text { Time dilation formula } \\
\mathrm{t}^{\prime}=((z / 2)+1)(z+1)^{.5} \mathrm{t} \\
\text { Equation } 4.1 \\
\text { alternative model time } \\
\text { dilation formula }\end{array}$ & $\begin{array}{l}\text { c. } \\
\text { The Change in distance } \\
\text { factor, D, } \\
\text { Also } 1 / \mathrm{D} \\
\text { Change in angular size } \\
\text { factor, } \sim \mathrm{D}^{-1}\end{array}$ & $\begin{array}{l}\text { d. } \\
\text { Complete brightness } \\
\text { Enhancement, in } \\
\text { Lumens } \\
\text { (Equation 11) }\end{array}$ \\
\hline Bin \# & $(z+1)$ & \multicolumn{3}{|c|}{ Type 1a supernovae and all cosmic entities } \\
\hline $\begin{array}{l}\text { 1) } \\
\text { 2) } \\
\text { 3) } \\
4) \\
5) \\
6) \\
7) \\
8) \\
9) \\
\text { 10) } \\
\text { 11) } \\
\text { 12) } \\
\text { 13) } \\
\text { 14) } \\
\text { 15) } \\
\text { 16) } \\
\text { 17) }\end{array}$ & $\begin{array}{l}1.0109 \\
1.0333 \\
1.1127 \\
1.1903 \\
1.2819 \\
1.3840 \\
1.4917 \\
1.6046 \\
1.7021 \\
1.8046 \\
1.9058 \\
2.0258 \\
2.1335 \\
2.2850 \\
2.4088 \\
2.5515 \\
2.6791\end{array}$ & $\begin{array}{l}1.0109 \\
1.0335 \\
1.1143 \\
1.1948 \\
1.2918 \\
1.4023 \\
1.5216 \\
1.6496 \\
1.7626 \\
1.8838 \\
2.0058 \\
2.1533 \\
2.2884 \\
2.4520 \\
2.6453 \\
2.8365 \\
3.0110\end{array}$ & $\begin{array}{l}1.0079^{-1} \\
1.0223^{-1} \\
1.0741^{-1} \\
1.1273^{-1} \\
1.1925^{-1} \\
1.2684^{-1} \\
1.3529^{-1} \\
1.4413^{-1} \\
1.5212^{-1} \\
1.6074^{-1} \\
1.6941^{-1} \\
1.7989^{-1} \\
1.8947^{-1} \\
2.0320^{-1} \\
2.1456^{-1} \\
2.2780^{-1} \\
2.3981^{-1}\end{array}$ & $\begin{array}{l}-.01184 \\
-.03584 \\
-.11809 \\
-.19689 \\
-.29094 \\
-.40004 \\
-.52304 \\
-.65884 \\
-.78463 \\
-.92403 \\
-1.0680 \\
-1.2454 \\
-1.4182 \\
-1.6456 \\
-1.8409 \\
-2.0666 \\
-2.2967\end{array}$ \\
\hline $\begin{array}{l}\text { Wave } \\
\text { " } z \text { " be } \\
2.0 \\
3.0 \\
4.0 \\
5.0 \\
6.0 \\
7.0 \\
8.0 \\
9.0 \\
10.0\end{array}$ & $\begin{array}{l}\text { engths }(z+1) \\
\text { yond type 1a SNe } \\
3.0000 \\
4.0000 \\
5.0000 \\
6.0000 \\
7.0000 \\
8.0000 \\
9.0000 \\
10.0000 \\
11.0000 \\
\end{array}$ & $\begin{array}{l}3.4641 \\
5.0000 \\
6.7082 \\
8.5732 \\
10.583 \\
12.728 \\
15.000 \\
17.393 \\
19.900 \\
\end{array}$ & $\begin{array}{l}2.7039^{-1} \\
3.6800^{-1} \\
4.6676^{-1} \\
5.6516^{-1} \\
6.1164^{-1} \\
7.5871^{-1} \\
8.5344^{-1} \\
9.4677^{-1} \\
10.3870^{-1} \\
\end{array}$ & $\begin{array}{c}-.2 .5027 \\
-.3 .7637 \\
-.5 .1140 \\
-6.2512 \\
-7.2185 \\
-7.9952 \\
-.8 .7631 \\
-9.5209 \\
-10.223 \\
\end{array}$ \\
\hline
\end{tabular}

Column "a." is the observed redshifted wavelengths which also represents the time dilation factor of the standard model. Column "b." is the calculated time dilation factor for the alternative model. Comparing the two it can be seen that at the farthest type 1a supernova observations to date, the length of the event should last about $12 \%$ longer in time. Column "c." is a factor which represents how much greater in distance the alternative model calculates than the Hubble formula. Its inverse is the calculation of what should accordingly be observed concerning the angular size of galaxies. Column "d." represents the calculated change in brightness for all cosmic entities (negative means brighter), primarily galaxies. For example for bin \#17, an increased factor of -2.2967 would calculate to about 8.2 times brighter. 
Table 2. Comparing Hubble-calculated distances with the Provisional and Final alternative distance calculations including predicted angular size calculations

\begin{tabular}{|c|c|c|c|c|}
\hline \multicolumn{2}{|c|}{$\begin{array}{c}\text { a. } \\
\text { Distances calculated by } \\
\text { Hubble formula, } \\
\text { where Hubble constant } \\
=66.34 \mathrm{~km} / \mathrm{sec} / \mathrm{Mpc} \\
\text { Megaparsecs }(\mathrm{Mpc})\end{array}$} & $\begin{array}{c}\text { b. } \\
\text { Alternative distances } \\
\text { calculated by } \\
\text { Provisional } \\
\text { Equation }(8) \\
\text { Mpc }\end{array}$ & $\begin{array}{c}\mathrm{c} . \\
\text { Alternative distances } \\
\text { calculated, by Final } \\
\text { Equation } \# 10 \mathrm{w} / \\
\text { additional factor }(z+1)^{5} \\
\mathrm{Mpc}\end{array}$ & $\begin{array}{c}\mathrm{d} . \\
\text { Columns c/a, } \\
\text { change in distance factor, } \\
\text { and } \\
\mathrm{a} / \mathrm{c} \text { Change in angular } \\
\text { size } \\
\text { factor or } \\
\mathrm{c} / \mathrm{a}^{-1} \\
\end{array}$ \\
\hline \multicolumn{5}{|c|}{ Bin \# \& redshifts same } \\
\hline 1) & 49.07 & 49.19 & 49.46 & $1.0079^{-1}$ \\
\hline 2) & 148.13 & 148.96 & 152.27 & $1.0223^{-1}$ \\
\hline 3) & 481.23 & 489.96 & 526.21 & $1.0741^{-1}$ \\
\hline 4) & 779.94 & 805.79 & 879.23 & $1.1273^{-1}$ \\
\hline 5) & $1,100.42$ & $1,158.90$ & $1,381.83$ & $1.1925^{-1}$ \\
\hline 6) & $1,420.10$ & $1,530.88$ & $1,941.50$ & $1.2684^{-1}$ \\
\hline 7) & $1,717.98$ & $1,912.18$ & $2,602.46$ & $1.3529^{-1}$ \\
\hline 8) & $1,992.10$ & $2,266.60$ & $2,871.14$ & $1.4413^{-1}$ \\
\hline 9) & $2,201.47$ & $2,566.98$ & $3,348.98$ & $1.5212^{-1}$ \\
\hline 10) & $2,397.52$ & $2,868.80$ & $3,853.86$ & $1.6074^{-1}$ \\
\hline 11) & $2,569.78$ & $3,153.50$ & $4,353.48$ & $1.6941^{-1}$ \\
\hline 12) & $2,750.15$ & $3,476.00$ & $4,947.35$ & $1.7989^{-1}$ \\
\hline 13) & $2,893.20$ & $3,753.03$ & $5,481.86$ & $1.8947^{-1}$ \\
\hline 14) & $3,068.51$ & $4,124.28$ & $6,234.29$ & $2.0320^{-1}$ \\
\hline 15) & $3,192.71$ & $4,413.21$ & $6,849.64$ & $2.1456^{-1}$ \\
\hline 16) & $3,317.97$ & $4,731.77$ & $7,558.23$ & $2.2780^{-1}$ \\
\hline 17) & $3,469.44$ & $5,088.18$ & $8,320.21$ & $2.3981^{-1}$ \\
\hline \multicolumn{5}{|c|}{$(z=)$} \\
\hline 2.00 & $3,617.86$ & $5,260.51$ & $9,782.33$ & $2.7039^{-1}$ \\
\hline 3.00 & $3,990.29$ & $6,638.06$ & $14,684.19$ & $3.6800^{-1}$ \\
\hline 4.00 & $4,174.46$ & $7,706.54$ & $19,484.50$ & $4.6676^{-1}$ \\
\hline 5.00 & $4,277.88$ & $8,579.54$ & $24,176.87$ & $5.6516^{-1}$ \\
\hline 6.00 & $4,341.44$ & $9,317.66$ & $28,767.03$ & $6.1164^{-1}$ \\
\hline 7.00 & $4,383.18$ & $9,957.06$ & $33,255.47$ & $7.5871^{-1}$ \\
\hline 8.00 & $4,412.03$ & $10,521.04$ & $37,654.16$ & $8.5344^{-1}$ \\
\hline 9.00 & $4,432.78$ & $11,025.53$ & $41,968.27$ & $9.4677^{-1}$ \\
\hline 10.00 & $4,448.19$ & $11,481.93$ & $46,203.16$ & $10.3870^{-1}$ \\
\hline
\end{tabular}

Table 2: Column "a." contains distances calculated by the standard Hubble formula. Column "b." contains calculated distances based upon the alternative model Provisional Equation (8). This equation is the basis for distances calculated for the subject supernova study. Column "c." is the complete distance equation. The additional factor $(z+1)^{.5}$ is equally compensated for by the final brightness equation so this factor does not show up as a change in brightnesses, but it shows up as a large decreased angular size of galaxies and other large observable cosmic entities. Column "d." is an indication of how much greater the calculated distances differ from the Hubble formula. Its inverse indicates how much smaller the angular size appears to be based upon Hubble calculated distances, but this size is directly proportional to calculations of the alternative model. 
Table 3. Comparing both alternative model brightnesses factors, separately and combined, based upon a changing time scale. These factors are combined with the inverse square law of E.M. radiation concerning luminosity

\begin{tabular}{cccc}
\hline a. & b. & c. & d. \\
Time Scale & Provisional Brightness & Final brightness factor & Entire brightness \\
(also as a Distance \& & Equation (6) & included; & $\begin{array}{c}\text { Enhancement equation of } \\
\text { Brightness factor) }\end{array}$ \\
Equation (5) & & $(z+1)$ & the alternative cosmologic \\
& & Added to Equation (6) & model \\
& & (Equation 11) \\
\hline
\end{tabular}

$\begin{array}{lrr} & \text { Bin \# } & \text { Timescale " } " \\ 1) & .023480 & .00000 \\ 2) & .070937 & -.00009 \\ 3) & .23115 & -.00156 \\ 4) & .37699 & -.00684 \\ 5) & .53736 & -.02004 \\ 6) & .70331 & -.04549 \\ 7) & .87046 & -.08674 \\ 8) & 1.02328 & -.14298 \\ 9) & 1.15096 & -.20441 \\ 10) & 1.27755 & -.27998 \\ 11) & 1.39563 & -.36444 \\ 12) & 1.52767 & -.47522 \\ 13) & 1.63983 & -.58289 \\ 14) & 1.78830 & -.74405 \\ 15) & 1.90249 & -.88178 \\ 16) & 2.02698 & -1.0447 \\ 17) & 2.18519 & -1.1947\end{array}$

Alternative model calculations of luminosities

$\begin{array}{ll}-.01184 & -.01184 \\ -.03575 & -.03584 \\ -.11653 & -.11809 \\ -.19005 & -.19689 \\ -.27090 & -.29094 \\ -.35455 & -.40004 \\ -.43630 & -.52304 \\ -.51586 & -.65884 \\ -.58022 & -.78463 \\ -.64405 & -.92403 \\ -.70358 & -1.0680 \\ -.77015 & -1.2454 \\ -.82668 & -1.4182 \\ -.90153 & -1.6456 \\ -.95909 & -1.8409 \\ -1.02185 & -2.0666 \\ -1.10198 & -2.2967\end{array}$

" $z$ " (redshifts)

$\begin{array}{ccc}2.0000 & 2.37743 & -1.4007 \\ 3.0000 & 3.00000 & -2.5652 \\ 4.0000 & 3.48288 & -3.6016 \\ 5.0000 & 3.87743 & -4.4954 \\ 6.0000 & 4.21102 & -5.2638 \\ 7.0000 & 4.49998 & -5.9787 \\ 8.0000 & 4.75487 & -6.6402 \\ 9.0000 & 4.98287 & -7.2523 \\ 10.0000 & 5.18913 & -7.8261\end{array}$

$\begin{array}{lr}-1.10198 & -.25027 \\ -1.19853 & -.37637 \\ -1.51237 & -.51140 \\ -1.75581 & -62512 \\ -1.95472 & -.72185 \\ -2.01651 & -.79952 \\ -2.12289 & -.87631 \\ -2.26856 & -.95209 \\ -2.39706 & -10.223\end{array}$

Table 3: Column "a." shows bin numbers and at the bottom, redshifts beyond distances where type 1a supernovas have been observed. Time periods in column "a." are regressive (progressively looking backward in time) and are based upon "doubling periods" concerning the size of matter in the past. See Equations 2, 3, 4, 5, $\& 6$ and related explanations. For example: at a redshift of 10, time frame 5.18913, matter would have been $\sim 3.17$ larger in diameter (the square root of $z+1$ ), which in this case is the square root of 11. In column "b." shows the progression of increased brightness based upon redshifts and directly proportional distances calculated by the alternative model. Column "c." shows the brightness addendum as a result of the addendum factor. This would not show up in either conventional or the alternative model concerning brightnesses. This is because in the standard model unobserved distances due to compensating brightnesses would be unobservable except by the decreased angular size of cosmic entities, Column "d.".

Note: For standard astronomy calculations the brightness adjustment factor is simply the inverse square law of light, where the alternative model proposes an additional brightness addendum factor needed to explain the brightness/luminosity of observations. 


\section{Further Discussion}

This discussion has focused on type 1a supernovae because there are strong reasons to believe that they are the standard candles that have long been sought after to provide valid distance indicators to distant galaxies. The distance and brightness equations of the alternative-model presented herein support this belief and therefore would apply to all cosmic entities. The distance equation supported by the supernova data might be described as a linear "Hubble law" directly proportional to the observed redshift, as first noted by Hubble when he recognized the proportional relationship between galactic redshifts and distances.

This distance equation and its linear relationship proportional to observed wavelengths, is based upon a theory of matter diminution. Matter slowly getting smaller over time would generally appear the same as galaxies moving away from us and each other. Differences between the alternative equations and the Hubble formula result from the proposition that matter, distances, and the speed of light, would appear to have been greater in the past. At the greatest distances, the observable universe would accordingly also be many times older than the Big Bang model could allow. The universe would accordingly be both Euclidean and static with galaxies aging in cycles while new galaxies would be continuously forming.

\subsection{Blue Shifted Galaxies as They Relate to the Subject Cosmological Model}

It is generally unknown that there have been thousands of observed blue shifted galaxies (Vanderlass, 2009; Gupta, 2013). These are galaxies presently moving toward us as explained similarly by both the standard and the alternative cosmological models. Thousands of galaxies slightly redshifted, thousands generally neutral having no redshifts, and also thousands of slightly Blueshifted galaxies have been observed in the local Virgo supercluster which we are a part of. Doppler shifting related to relative motion, is the simplest explanation of these observations. Blue shifting can be produced in front of our relative motion and redshifting of galaxies behind our relative motion.

First to be considered regarding relative motions is the orbital velocity of the Earth around the sun, next the orbital velocity of our solar system around our galaxy, next the orbital motion of our galaxy within our Local Group, and then the local group orbits within the Virgo supercluster. It has been asserted, although still controversial, that the group of local superclusters, including our Virgo supercluster, is moving at a great velocity relative to the micro-wave background and the over-all distant background of galaxies. This has been dubbed the "Dark Flow," which is supposedly toward a "Great Attractor". That would be only a part of the overall relative motions involved for calculations. Most other galaxies would also have similar relative motions by orbiting within their local groups, within their supercluster, and could have even greater orbital or linear motions like the dark flow at an even grander scale, relative to the overall background of galaxies.

This is probably the primary reason why that within our Local Group and our local supercluster Virgo, redshifts are not reliable indicators of galactic distances. This is also why other distance indicators are used to determine distances of entities within our supercluster. These distance measurement methods are collectively called The Distance Ladder. Within the local Virgo Supercluster all relative motions could cancel out each other since they are additive. Relative motions including all of our own, plus those of observed galaxies, have produced thousands of blue-shifted galaxies from our perspective. The largest Blueshifted galaxies have been found to be some of the closest in our Local Group and supercluster. Several thousand slightly blue-shifted galaxies have been observed overall, primarily within our Virgo supercluster. The closest large galaxy Andromeda is the most well-known blue-shifted galaxy from our perspective.

In any non-expanding-universe cosmological model, like the subject model the Pan Theory (Noble, 2012), the appearance of Blue-shifted galaxies would be expectedly more prevalent. Only at distances and timeframes beyond our supercluster could the increased size of matter with longer wavelengths, accordingly overcome relative motions that would otherwise produce equal quantities of redshifting and blueshifting.

In both models, whether expanding space or matter getting smaller, additional gravity would seem to be needed to hold solar systems, galaxies, galaxy groups and superclusters, together to enable them to maintain their observed forms for a long period of time - or to have formed in the first place. This is where a different theory of gravity might better explain observed reality.

\subsection{Reference to Dark Matter}

Brief mention: The subject cosmological model proposes that dark matter does not exist. It instead proposes an alternative theory of gravity, generally something like MOND gravity (Wikepedia, Modified Newtonian dynamics, 2012), but with what is believed to be a strong theoretical basis. Additional refinement concerning the final determinations of equations concerning values of constants and balancing variables has not been finally 
determined. A study with adequate stellar, galaxy and cluster-motion data will be needed by the authors to further refine these preliminary equations.

Note: Readers may still have unanswered questions regarding the subject cosmological model that were discussed in this study. For these questions contact the authors through the noted reference e-mail address herein, concerning corrections, questions, etc. as noted below in "Responses".

For all questions concerning the alternative cosmological model which may or may not be directly related to this study, such as dark matter mentioned above, readers could also look for answers at the related website the Pan Theory (Noble, 2012) or contact the authors through the e-mail address given herein, anytime following the publication of this paper.

\section{Summary of Reasons Why This Paper and the Alternative Cosmological Model and Equations Should Seriously Be Considered and Evaluated}

1) Type 1a supernovae observations: The primary reason for this consideration might be that the consensus of cosmologists, including the authors, considers type 1a supernovas as standard candles of cosmic distances. Because of their brightnesses nearly all agree, including the authors, that supernovas closer than a redshift of $(z$ $=.5$ ) are farther away than what the Hubble formula calculates (Wikipedia, Accelerated Expansion, 2013). The authors believe that an inaccurate Hubble formula along with a non-expanding universe model, is a far better explanation than the universe having to first accelerate its expansion superluminally (Inflation), then to decelerate this expansion, then again to accelerate expansion again (dark energy) during a timeframe at a distance determined by a redshift of $z \sim .5$.

Conclusion 1. An incorrect distance formula seems far more likely than the universe having to perform multiple tasks explained by proposing an additional unknown, unobserved new force like dark energy.

2) Observation angels: The observed angular sizes of galaxies are considered an anomaly in standard cosmology as discussed in detail above. The observed angular sizes of galaxies are off by many factors of magnitude and for this require an ad hoc hypothesis of galaxy evolution to explain galaxies being much more condensed in the past. The alternative model herein exactly fits the observed angular sizes of galaxies predicted for normal galaxy evolution.

Conclusion 2. A model of the universe where the model's equations easily explain the observed angular sizes and observed brightnesses of all cosmic entities, seems far more likely than a universe requiring a number of ad hoc hypothesis (unexpected based upon theory) such as dark energy, dark matter, and other hypothesis such as the seemingly ad hoc galaxy evolutionary processes required to explain the Tolman test.

3) The Tolman Test: The Tolman surface brightness test, was conceived in the 1930 by Richard Tolman to compare cosmological models.

The Tolman test compares the surface brightness of galaxies as they relate to redshifts (designated as $z$ ), to determine as to whether the universe is expanding or not. The initial results of the studies of those initial times seemed contrary to an expanding universe.

The logic goes like this: In a simple flat, static universe, light intensity drops inversely with the square of the distance from its source. The apparent area of the object also drops inversely with the square of the distance from the source.

"In an expanding universe, however, there are two (additional) effects that reduce the power detected coming from distant objects. First, the rate at which photons are received is reduced because each photon has to travel a little farther than the one before. Second, the energy of each photon observed is reduced by the redshift. At the same time, distant objects appear larger than they really are because the photons observed were emitted at a time when the object was closer. Adding (all) these effects together, the surface brightness in a simple expanding universe (flat geometry and uniform expansion over the range of redshifts observed) should decrease with the fourth power of $(1+z), 1 /(1+z) 4$ " (Wikipedia, Tollman signal, 2001).

Also according to the Big Bang model the universes' density should have steadily decreased over time based upon the expansion of space. No consensus opinions concerning broad-scale observations or studies have been made regarding the density of galaxies per volume, for any timeframe in the past, having been greater than in the present except by inferences of the Big Bang model.

The additional factor, energy being reduced by redshifts, would be the same for both the standard model and the proposed alternative model, so the primary differences between these models would be the decreased brightnesses produced from an expanding universe based upon the standard model. 
"To date, the best investigation of the relationship between surface brightness and redshift was carried out using the $10 \mathrm{~m}$ Keck telescope to measure nearly a thousand galaxies" redshifts and the $2.4 \mathrm{~m}$ Hubble Space Telescope to measure those galaxies' surface brightness.

"The exponent found (was) not 4 as expected in the simplest expanding (universe) model, but (instead was) 2.6 (to) 3.4, depending on the frequency band (being evaluated)" (parenthesis added) (Wikipedia, Tollman signal, 2001).

In a non- expanding universe like the alternative model, the Tolman Test would reduce to simply the inverse square law of light, luminosity distance, plus the decrease in brightness due to the decreased frequency of E.M. radiation without a forth power exponent. This would result in a constant brightness ratio of $1 /(1+z)^{3}$ based upon the alternative model distance and brightness equations.

Based upon observations and distances calculated by the Hubble formula, brightness ratios begin at about $z \sim 1 /$ $(1+z)^{3}$ for the closest supernovae. Brightnesses then decrease to their dimmest extent of $\sim 1 /(1+z)^{3.4}$ at a redshift of $\sim z=.5$; then brightnesses increase again to a ratio of $1 /(1+z)^{2.6}$ at the farthest observed redshifts of type 1a supernova concerning the data used/ available in this study. This was at a redshift of $z \sim 1.679$ (a wavelength of 2.679). Beyond this redshifts according to predictions of the alternative model concerning Hubble calculated distances and brightnesses concerning all types of supernovae, should progressively and steadily increase in their brightnesses based upon Hubble formula calculations, to the edge of the observable universe, as this ratio would continue to decrease. This would not be the case if distances were much greater than the Hubble formula would calculate such as distances and brightnesses determined by the alternative equations.

The Tolman Test, based upon the alternative model equations, would seem to indicate a static, non-expanding universe. The same test using the Big Bang model with the Hubble formula, does not meet the requirements of the Tolman Test. To explain the results of this test, ad hoc galaxy evolutionary hypothesis such as those proposed by Bruzual and Charlot (Wikipedia, Tolman Signal, 2001) are thought to be needed to explain these unexpected brightnesses of galaxies at the greatest distances. For supernovas additional hypothesis concerning supernovae evolution have been proposed to explain the unexpected brightnesses observed concerning the most distant cosmic entities (Foley et al., 2008).

Conclusion 3. A cosmological model that passes cosmological tests concerning theory and observation, is more likely to be valid than one that fails the same tests unless additional untestable ad hoc hypothesis are added. This may be the case concerning the Tolman test contradicting an expanding universe, or the case for Hubble calculated distances being contradicted by the inverse square law of light concerning cosmic entities, unless the dark energy hypothesis is added for justification.

\section{General Conclusions and Applications}

This paper presents a study of type 1a supernovae data comparing the standard Hubble distance formula and Luminosity Distances (Wikipedia, Luminosity Distance, 2013) with an alternative cosmological model with its own equations. The result of the alternative equations seems to effectively explain the observed brightness-versus-distance trend of type 1a supernovae based upon a linear, "static" universe model as represented by a very good standard candle line. According to the analyses herein the reason why dark energy was postulated in the first place was seemingly because of the miscalculation of distances by the Hubble formula. The alternative model and its equations, on the other hand, indicate no need for the theoretical complications of the accelerated and/or decelerated expansion of the universe and dark energy. The alternative cosmological model proposes that the observable universe is not expanding, that space itself does not expand or warp. It proposes that the most distant entities, galaxies, quasars, and supernovae, currently observable having wavelengths of roughly $z=1.5$ or greater, are at distances much greater than the present Hubble formula could allow.

The alternative cosmological model presented here is called the Pan Theory, the details of which include answers to many "why" and "how" questions, proposed tests of the model, its implications and predictions. All can be found at the related website (Noble, Pan Theory cosmology, 2012). For example such a question and extended answers relate to why matter accordingly becomes smaller over time.

The plots and tabulated data (included Figures) comparing the alternative model to the conventional one, are the result of a best-fit analysis by the authors after extended study. Based on the alternative cosmological model concerning the diminution of matter and proceeding from that "first principle," the subject equations were derived. It should be noted that the alternative equations in this paper contradict the Big Bang model and cannot be derived from its premises. 


\subsection{Applications/Implications}

Those who wish to try out these proposed alternative-model distance and luminosity, or time dilation equations on real observations, Equations 10, 11, and 12 respectively, may also find solutions to other problems in cosmology. One such problem that has been discussed herein is the anomalous angular sizes concerning cosmic redshifted entities, primarily galaxies and quasars (LopezCordera, 2010; Unzicker; 2010). Many astronomers have noted that the observation angles of galaxies are generally inversely proportional to their observed redshifts based upon Hubble calculated distances. To explain these anomalies some mainstream theorists have proposed that galaxies collectively underwent a vast unpredicted universe-scale evolution (Epinat et al., 2009).

Correctly determined/calculated distances could also solve other perceived cosmological problems such as why distant cosmological entities such as quasars seem to be so bright but their brightness variations are not time-dilated, unlike the EM radiation resulting from supernova explosions.

It is hoped that others will try to recreate the subject study and analyses of type 1a supernovas based upon the same data set, an improved or updated data set, and the subject cosmological model to justifiably criticize, correct, confirm, or add improvements to these conclusions.

The distance and brightness equations offered provide an explanation for observed angular sizes of cosmic entities (LopezCordera, 2010; Unzicker, 2010) without requiring cosmic evolution, using one of the simplest possible cosmological models of a Euclidean non-expanding universe, with explanations consistent with observed redshifts. It is further hoped that this alternative interpretation, if confirmed, will solve the problem of dark energy by showing it to be a construct of a sub-optimal cosmological model akin to adding epicycles in geocentric theory.

Responses:

Please contact the author Forrest Noble at pantheory.org@gmail.com. He will be very happy to answer any questions, consider corrections, and comments. If you are interested in testing these equations, if you have new insights or need additional insights into this alternative cosmological model and equations, the authors are willing to discuss this.

\section{References}

Brizual, \& Charlot. (2006). Fitting the spectral energy distributions of galaxies. Retrieved from http://www.sedfitting.org/SED08/Models.html

Conley, A., Guy, J., Sullivan, M., Regnault, N., Astier, P., Balland, C., ...Walker, E. S. (2011). Measuring the Curvature of the Universe by Measuring the Curvature of the Hubble Diagram. Retrieved from http://www.astro.ucla.edu/ wright/sne_cosmology.html

Das, P. K. (1997). Quasars in variable mass hypothesis. Journal of Astrophysics and Astronomy, 18(4), 435-440. http://dx.doi.org/10.1007/BF02709334

Epinat, B., Amram, P., Balkowski, C., \& Macelin, M. (2010). Evidence for strong dynamical evolution in disc galaxies through the last 11 Gyr. GHASP VIII - a local reference sample of rotating disc galaxies for high-redshift studies. Monthly Notices of the Royal Astronomical Society, 401(4), 2113-2147. http://dx.doi.org/10.1111/j.1365-2966.2009.15688.x

Foley, R. J., Filippenko, A. V., Aguilera, C., Becker, A. C., Blondin, S., Challis, P., ... Zenteno, A. (2008). Constraining Cosmic Evolution of Type Ia Supernovae. The Astrophysical Journal, 684(1), 68. http://dx.doi.org/10.1086/589612

Gupta, S. N. P. (2013). Dynamic universe model. Retrieved from http://vaksdynamicuniversemodel.blogspot.com/2012/03/mathematical-prediction-of-existence-of.html

Hubblesite. (2013). Dark Energy, fate of universe. Retrieved from http:/hubblesite.org/hubble_discoveries/dark_energy/de-fate_of_the_universe.php

Kowalski, M., Rubin, D., Aldering, G., Agostinho, R. J., Amadon, A., Amanullah, R., ... Yun, J. L. (2008). Improved Cosmological Constraints from New, Old, and Combined Supernova Data Sets. The Astrophysical Journal, 686(2), 749. http://dx.doi.org/10.1086/589937

Las Cumbres Observatory. (2012). Redshifts. Retrieved from http://lcogt.net/education/article/redshift

López-Corredoira, M. (2010). Angular size test on the expansion of the Universe. Retrieved from http://arxiv.org/abs/1002.0525 
Lubin, L. M., \& Sandage, A. (2001). The Tolman Surface Brightness Test for the Reality of the Expansion. IV. A Measurement of the Tolman Signal and the Luminosity Evolution of Early-Type Galaxies. The Astronomical Journal, 122(3), 1084-1103.

NASA. (2013). Re: flat universe. Retrieved from http://map.gsfc.nasa.gov/universe/uni_shape.html

Noble, F. (2012). Pan Theory cosmology. Retrieved from http://www.pantheory.org/Pages/index2.php

Unzicker, A. (2007). A Look at the Abandoned Contributions to Cosmology of Dirac, Sciama and Dicke. Retrieved from http://arxiv.org/abs/0708.3518

Unzicker, A., \& Fabian, K. (2010). New Directions in Modern Cosmology. Leiden. Retrieved from www.vom-urknall-zum-durchknall.de http://www.lorentzcenter.n1/lc/web/2010/412/presentations/Unzicker.pdf

Vanderlass, K. (2009/2012). Distribution Blue Shifted Galaxies; reference NASA/IPAC Extragalactic Database (NED). Retrieved from http://fittedplane.blogspot.com/2009/12/blue-shifted-galaxies-there-are-more.html

Wikibooks, Theoretical Physics. (2013). Retrieved from http://en.wikibooks.org/wiki/Introduction_to_Theoretical_Physics

Wikipedia, Accelerating Universe. (2013). Retrieved from http://en.wikipedia.org/wiki/Accelerating_universe Wikipedia, Dark Energy. (2013). Retrieved from http://en.wikipedia.org/wiki/Dark_energy

Wikipedia, Dark Flow, Great Attractor. (2013). Retrieved from http://en.wikipedia.org/wiki/Dark_flow Wikipedia, De Sitter Relativity. (2013). Retrieved from http://en.wikipedia.org/wiki/De_Sitter_relativity Wikipedia, Luminosity Distance. (2013). Retrieved from http://en.wikipedia.org/wiki/Luminosity_distance Wikipedia, Metric Expansion. (2013). Retrieved from http://en.wikipedia.org/wiki/Metric_expansion_of_space Wikipedia, Robert H. Dicke. (1956). Retrieved from http://en.wikipedia.org/wiki/Robert_H._Dicke Wikipedia, Surface Brightness. (2013). Retrieved from http://en.wikipedia.org/wiki/Surface_brightness Wikipedia, Tolman Signal. (2013) Retrieved from http://en.wikipedia.org/wiki/Tolman_surface_brightness_test

\section{Copyrights}

Copyright for this article is retained by the author(s), with first publication rights granted to the journal.

This is an open-access article distributed under the terms and conditions of the Creative Commons Attribution license (http://creativecommons.org/licenses/by/3.0/). 